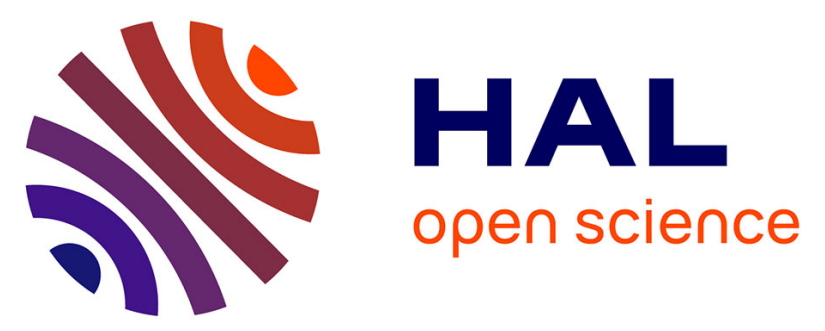

\title{
Estimation of the caesium-137 source term from the Fukushima Daiichi nuclear power plant using a consistent joint assimilation of air concentration and deposition observations
}

Victor Winiarek, Marc Bocquet, Nora Duhanyan, Yelva Roustan, Olivier Saunier, Anne Mathieu

\section{To cite this version:}

Victor Winiarek, Marc Bocquet, Nora Duhanyan, Yelva Roustan, Olivier Saunier, et al.. Estimation of the caesium-137 source term from the Fukushima Daiichi nuclear power plant using a consistent joint assimilation of air concentration and deposition observations. Atmospheric Environment, 2014, 82, pp.268-279. 10.1016/j.atmosenv.2013.10.017 . hal-00907484v2

\author{
HAL Id: hal-00907484 \\ https://hal.inria.fr/hal-00907484v2
}

Submitted on 26 Nov 2013

HAL is a multi-disciplinary open access archive for the deposit and dissemination of scientific research documents, whether they are published or not. The documents may come from teaching and research institutions in France or abroad, or from public or private research centers.
L'archive ouverte pluridisciplinaire HAL, est destinée au dépôt et à la diffusion de documents scientifiques de niveau recherche, publiés ou non, émanant des établissements d'enseignement et de recherche français ou étrangers, des laboratoires publics ou privés. 


\title{
Estimation of the caesium-137 source term from the Fukushima Daiichi nuclear power plant using a consistent joint assimilation of air concentration and deposition observations
}

\author{
Victor Winiarek $^{\mathrm{a}, \mathrm{b}}$, Marc Bocquet ${ }^{\mathrm{a}, \mathrm{b}}$, Nora Duhanyan $^{\mathrm{a}}$, Yelva Roustan ${ }^{\mathrm{a}}$, Olivier Saunier ${ }^{\mathrm{c}}$, Anne Mathieu $^{\mathrm{c}}$ \\ ${ }^{a}$ Université Paris-Est, CEREA, joint laboratory École des Ponts ParisTech and EDF RED, Champs-sur-Marne, France \\ ${ }^{b}$ INRIA, Paris Rocquencourt research centre, France \\ ${ }^{c}$ Institut de Radioprotection et de Sûreté Nucléaire (IRSN), PRP-CRI, SESUC, BMTA, Fontenay-aux-Roses, 92262, France
}

\begin{abstract}
Inverse modelling techniques can be used to estimate the amount of radionuclides and the temporal profile of the source term released in the atmosphere during the accident of the Fukushima Daiichi nuclear power plant in March 2011. In Winiarek et al. (2012b), the lower bounds of the caesium-137 and iodine-131 source terms were estimated with such techniques, using activity concentration measurements. The importance of an objective assessment of prior errors (the observation errors and the background errors) was emphasised for a reliable inversion. In such critical context where the meteorological conditions can make the source term partly unobservable and where only a few observations are available, such prior estimation techniques are mandatory, the retrieved source term being very sensitive to this estimation.

We propose to extend the use of these techniques to the estimation of prior errors when assimilating observations from several data sets. The aim is to compute an estimate of the caesium-137 source term jointly using all available data about this radionuclide, such as activity concentrations in the air, but also daily fallout measurements and total cumulated fallout measurements. It is crucial to properly and simultaneously estimate the background errors and the prior errors relative to each data set. A proper estimation of prior errors is also a necessary condition to reliably estimate the a posteriori uncertainty of the estimated source term. Using such techniques, we retrieve a total released quantity of caesium-137 in the interval $11.6-19.3 \mathrm{PBq}$ with an estimated standard deviation range of $15-20 \%$ depending on the method and the data sets. The "blind" time intervals of the source term have also been strongly mitigated compared to the first estimations with only activity concentration data.
\end{abstract}

This article has been published in Atmospheric Environment with the reference:

Winiarek, V., Bocquet, M., Duhanyan, N., Roustan, Y., Saunier, O., Mathieu, A., 2014. Estimation of the caesium-137 source term from the Fukushima Daiichi nuclear power plant using a consistent joint assimilation of air concentration and deposition observations. Atmos. Env. 82. 268-279.

Keywords: Data assimilation, Atmospheric dispersion, Fukushima accident, Source estimation

\section{Introduction}

\subsection{The Fukushima Daiichi accident}

On March 11, 2011, 05:46 UTC, a magnitude 9.0 $\left(\mathrm{M}_{\mathrm{w}}\right)$ undersea megathrust earthquake occurred in the Pacific Ocean and an extremely destructive tsunami hit the Pacific coast of Japan approximately one hour later. These events caused the automatic shut-down of

Email address: bocquet@cerea.enpc.fr (Marc Bocquet)
4 power plants in Japan. Diesel backup power systems should have sustained the reactors cooling process. In Fukushima Daiichi these backup devices were unfortunately inoperative mainly because of the damages caused by the tsunami.

The Fukushima Daiichi NPP has six nuclear reactors. At the time of the earthquake, reactor 4 had been defuelled and reactors 5 and 6 were in a cold shut-down for planned maintenance.

In the hours that followed, the situation quickly be- 
came critical. Reactors 1, 2 and 3 experienced at least partial meltdown and hydrogen explosions. Additionally, fuel rods stored in pools in each reactor building began to overheat as water levels in the pools dropped.

All these events caused a massive discharge of radioactive materials in the atmosphere. The total quantities of released radionuclides as well as the time evolution of these releases have to be estimated in order to assess the sanitary and environmental impact of the accident.

Mathieu et al. (2012) used core inventories and insitu data (pressure and temperature measurements in the reactors and $\gamma$-dose rates in the NPP) as well as first available observations over Japan to build a source term, which was used in Korsakissok et al. (2013) to assess the high $\gamma$-dose rates zones at a local scale. Chino et al. (2011) used a few activity concentration measurements in the air to calibrate the magnitude of some identified releases of ${ }^{131} \mathrm{I}$ and ${ }^{137} \mathrm{Cs}$. Katata et al. (2012) and Terada et al. (2012) updated these estimations as new data were available. Stohl et al. (2012) performed an inverse modelling estimation using activity concentrations in the air, as well as deposition data at a global scale to estimate the release of ${ }^{133} \mathrm{Xe}$ and ${ }^{137} \mathrm{Cs}$. However their estimation was shown to be quite sensitive to the prior information on the source used in the inversion. At the same time, Winiarek et al. (2012b) proposed a method to properly estimate the prior errors to perform inverse modelling using activity concentrations in the air. They applied it to estimate the source terms of ${ }^{131} \mathrm{I}$ and ${ }^{137} \mathrm{Cs}$.

\subsection{Objectives and outline}

In Winiarek et al. (2012b) we emphasised the importance of a proper estimation of prior errors in the inverse modelling algorithm. We proposed new methods to estimate two hyper-parameters: the variance of observation errors and the variance of background errors, assuming that all observation errors have the same variance. This assumption is acceptable when using only one type of data in the algorithm. On the other hand the general lack of data in accidental situations stresses the need for algorithms that would use all available data in the same inversion. The first objective of this paper is to extend the methods proposed in Winiarek et al. (2012b) to the simultaneous estimation of prior errors when using different data sets in the inversion. The second objective is to apply these methods to the challenging reconstruction of the caesium-137 source term of the Fukushima Daiichi accident, as well as to obtain an objective uncertainty on this estimation.

In Section 2 we briefly recall the methodology for the inverse modelling of accidental releases of pollutants.
The algorithm is sensitive to the statistics of the errors so that we propose methods based on the maximum likelihood principle to estimate these errors. This general maximum likelihood scheme takes into account the positivity of the source and the presence of several types of data.

In Section 3 we apply this new method to the reconstruction of the atmospheric release of ${ }^{137} \mathrm{Cs}$ from the Fukushima Daiichi power plant. The inversions are computed using three data sets: activity concentrations in the air, daily measurements of deposited material and total cumulated deposition. The new source terms are discussed and compared to earlier estimations. The posterior uncertainties of the retrieved sources are also computed.

Results are summarised and conclusions are given in Section 4.

\section{Methodology}

\subsection{Inverse modelling of accidental releases and esti- mation of errors}

To reconstruct the source term of an accidental release of a pollutant into the atmosphere, inverse modelling techniques are a powerful alternative to a trial and error approach with direct numerical models, which is still widely used in such situations. Inverse modelling techniques can objectively estimate the source term using the information content of an observation set and a numerical model that simulates the dispersion event. The atmospheric transport model (ATM), which is linear in our case for gaseous and particulate matter, provides the relationship between the source term and the observation set through the source-receptor equation

$$
\boldsymbol{\mu}=\mathbf{H} \sigma+\boldsymbol{\epsilon},
$$

where $\boldsymbol{\mu}$ in $\mathbb{R}^{d}$ is the measurement vector, $\boldsymbol{\sigma}$ in $\mathbb{R}^{N}$ is the source vector, and $\mathbf{H}$ is the Jacobian matrix of the transport model which incorporates the observation operator as well. The vector $\boldsymbol{\epsilon}$ in $\mathbb{R}^{d}$, called the observation error in this article, represents the instrumental errors, the representativeness errors and a fraction of model error altogether.

In an accidental context the number of observations is very often limited. Specific meteorological conditions, like during the Fukushima accident, can also lead to a weak observability of a part of the source term. In these cases, the source-receptor relationship defined by Eq. (1) constitutes an ill-posed inverse problem (Winiarek et al., 2011). 
One solution to deal with the lack of constraints is to implement parametric methods where the source to retrieve is reduced to a very limited number of parameters. The inverse problem is de facto regularised and it can be solved using different techniques, such as stochastic sampling techniques which allow to access the parameters posterior distributions (Delle Monache et al., 2008; Yee et al., 2008). Nevertheless if the true source does not match the parametric model, the inversion may fail or yield a meaningless solution.

Another option is the use of non-parametric methods to retrieve a general source field. This field can be discretised, for example to match the model discretisation, but the number of variables remains large and may still be larger than the number of observations in the data set. The non-parametric approach is robust and flexible since no strong a priori assumptions are made on the source but it has its own constraints. If Gaussian statistics are assumed for observation errors, the inversion relies on the minimisation of the cost function

$$
\mathcal{L}(\boldsymbol{\sigma})=\frac{1}{2}(\boldsymbol{\mu}-\mathbf{H} \boldsymbol{\sigma})^{\mathrm{T}} \mathbf{R}^{-1}(\boldsymbol{\mu}-\mathbf{H} \boldsymbol{\sigma}),
$$

where $\mathbf{R}$ is the observation error covariance matrix: $\mathbf{R}=\mathrm{E}\left[\boldsymbol{\epsilon} \boldsymbol{\epsilon}^{\mathrm{T}}\right]$, where $\boldsymbol{\epsilon}$ has been defined by Eq. (1). One simple choice is to neglect correlations between observation errors and to take $\mathbf{R}$ diagonal. If all the observations are of the same type, one can even assume that $\mathbf{R}=r^{2} \mathbf{I}_{d}, r^{2}$ being the variance of the observation errors $\left(\boldsymbol{I}_{d}\right.$ is the identity matrix in $\mathbb{R}^{d \times d}$ ). With a low number of observations and/or a poor observability of several source term parts, the minimisation of Eq. (2) gives infinitely many solutions. One needs to regularise the inverse problem, usually by adding a Tikhonov term in the cost function:

$$
\begin{aligned}
\mathcal{L}(\sigma)= & \frac{1}{2}(\boldsymbol{\mu}-\mathbf{H} \boldsymbol{\sigma})^{\mathrm{T}} \mathbf{R}^{-1}(\boldsymbol{\mu}-\mathbf{H} \boldsymbol{\sigma}) \\
& +\frac{1}{2}\left(\boldsymbol{\sigma}-\boldsymbol{\sigma}_{b}\right)^{\mathrm{T}} \mathbf{B}^{-1}\left(\boldsymbol{\sigma}-\boldsymbol{\sigma}_{b}\right) .
\end{aligned}
$$

The solution of the inverse problem is now unique, but two additional (vector and matrix) parameters have been introduced: $\sigma_{b}$ is the first guess for the source (or background term) and $\mathbf{B}$ is the background error covariance matrix: $\mathbf{B}=\mathrm{E}\left[\left(\boldsymbol{\sigma}-\boldsymbol{\sigma}_{b}\right)\left(\boldsymbol{\sigma}-\boldsymbol{\sigma}_{b}\right)^{\mathrm{T}}\right]$.

In an accidental situation and particularly in the Fukushima accident context, the choice of $\boldsymbol{\sigma}_{b}=\mathbf{0}$ is relevant because (i) many of the parameters are likely to be zero (ii) it guarantees an independent estimate (iii) it avoids the risk of an inversion crime, since most of the first guess built by physics models are eventually calibrated using early observations. One can refer to
Bocquet (2005); Davoine and Bocquet (2007); Winiarek et al. (2012b) for extended discussions on this choice.

Still in an accidental context, off-diagonal terms in $\mathbf{B}$ are often negligible. For example the $\gamma$-dose measurements made in the Fukushima nuclear power plant (NPP) indicate that the source term is probably composed of uncorrelated events ${ }^{1}$. This is the reason why we take $\mathbf{B}=m^{2} \mathbf{I}_{N}$ in this article.

As it was shown in the Chernobyl case in Davoine and Bocquet (2007) or in the Fukushima case in Winiarek et al. (2012b) or Stohl et al. (2012), the retrieved source is very sensitive to the matrices $\mathbf{R}$ and $\mathbf{B}$, hence to the two hyper-parameters $r$ and $m$. This is the reason why hyper-parameters estimation techniques are required. In Davoine and Bocquet (2007); Krysta et al. (2008); Saide et al. (2011), the L-curve technique of Hansen (1992) was successfully used to estimate the ratio $r / m$ or both parameters. In the recent years several methodological developments in the data assimilation for weather forecast have focused on the estimation of the hyper-parameters of the prior errors. They are mostly based on either cross-validation technique or on the maximum likelihood principle (e.g. Mitchell and Houtekamer, 1999; Chapnik et al., 2004, 2006; Anderson, 2007; Li et al., 2009). These techniques have also been implemented in the context of atmospheric chemistry inverse modelling, using for example the $\chi^{2}$ criterion (Ménard et al., 2000; Elbern et al., 2007; Davoine and Bocquet, 2007), the maximum likelihood principle (Michalak et al., 2004), or statistical diagnostics (Schwinger and Elbern, 2010).

In Winiarek et al. (2012b) we implemented several methods for the estimation of $r$ and $m$. One of these methods relies on the L-curve coupled with a $\chi^{2}$ criterion. Another one is based on the maximum likelihood principle and takes into account without approximation the positivity of the source. This study only exploited activity concentrations in the air. In the case where several types of data are used in the inversion algorithm, the number of hyper-parameters to estimate increases. One can for instance try to estimate one value of the variance of observation errors for each data set, that is an $r^{2}$ attached to each data set. This simultaneous estimation of prior errors is the methodological objective of this article.

\footnotetext{
${ }^{1}$ http://www.tepco.co.jp/en/nu/monitoring/index-e.
} html 


\subsection{Prior errors statistics and cost function minimisa-} tion

The observation errors defined by Eq. (1) are assumed normally distributed, with a probability density function (pdf):

$$
p_{e}(\boldsymbol{\epsilon})=\frac{e^{-\frac{1}{2} \boldsymbol{\epsilon}^{\mathrm{T}} \mathbf{R}^{-1} \boldsymbol{\epsilon}}}{\sqrt{(2 \pi)^{d}|\mathbf{R}|}}
$$

where $|\mathbf{R}|$ is the determinant of the observation error covariance matrix $\mathbf{R}$. $\mathbf{R}$ is assumed diagonal (it is an approximation as model errors may introduce some correlations). Besides, it is assumed that the errors made on observations of the same type have the same variance, so that if $\mathbf{R}_{i}$ represents the sub-block of $\mathbf{R}$ relative to the data set $i$, one has $\mathbf{R}_{i}=r_{i}^{2} \mathbf{I}_{d_{i}}$, where $d_{i}$ is the number of observations in data set $i\left(\sum_{i=1}^{i=N_{d}} d_{i}=d, N_{d}\right.$ is the number of different data sets) and $r_{i}^{2}$ is the corresponding error variance. Note that due to the limited number of observations and in order to avoid a too severely underconstrained problem, it is essential to keep the number of hyper-parameters that define $\mathbf{R}$ as small as possible $\left(N_{d}\right.$ hyper-parameters in our case: $\left.\left(r_{i}\right)_{1 \leq i \leq N_{d}}\right)$. For instance, an approach such as the one put forward by Desroziers et al. (2005) or Schwinger and Elbern (2010) is likely to be unaffordable in this accidental context.

As far as background errors are concerned, we could also use Gaussian statistics. The advantages of this choice would be analytical solutions for both the source term estimation and the related uncertainty based on the Best Linear Unbiased Estimator (BLUE) theory. However, such a Gaussian assumption could lead to negative values in the retrieved source term, because of the lack of sufficient observations to constrain the source term. To avoid non-physical results, truncated normal statistics for background errors are considered, enforcing the positivity of the retrieved source term. It is known to provide valuable information to the data assimilation system (Bocquet et al., 2010) and was successfully used in the context of the Fukushima Daiichi accident (Winiarek et al., 2012b). The corresponding pdf of this normalised truncated normal distribution reads in the general case

$$
\left\{\begin{array}{rll}
\text { if } \quad \boldsymbol{\sigma} \geq \mathbf{0} \quad p(\boldsymbol{\sigma})= & \left(\int_{\boldsymbol{s} \geq \mathbf{0}} e^{-\frac{1}{2}\left(\boldsymbol{s}-\boldsymbol{\sigma}_{b}\right)^{\mathrm{T}} \mathbf{B}^{-1}\left(\boldsymbol{s}-\boldsymbol{\sigma}_{b}\right)} \mathrm{d} \boldsymbol{s}\right)^{-1} \\
& \times e^{-\frac{1}{2}\left(\sigma-\sigma_{b}\right)^{\mathrm{T}} \mathbf{B}^{-1}\left(\boldsymbol{\sigma}-\boldsymbol{\sigma}_{b}\right)} \\
& \\
\text { otherwise } \quad p(\boldsymbol{\sigma})= & 0 .
\end{array}\right.
$$

The normalisation factor can be simplified in our case where $\mathbf{B}$ is diagonal and $\sigma_{b}=\mathbf{0}$ to yield the following
semi-Gaussian pdf:

$$
\begin{cases}\text { if } \quad \boldsymbol{\sigma} \geq \mathbf{0} & p(\boldsymbol{\sigma})=\frac{e^{-\frac{1}{2} \sigma^{\mathrm{T}} \mathbf{B}^{-1} \boldsymbol{\sigma}}}{\sqrt{(\pi / 2)^{N}|\mathbf{B}|}} \\ \text { otherwise } & p(\boldsymbol{\sigma})=0,\end{cases}
$$

where $|\mathbf{B}|$ is the determinant of the background error covariance matrix $\mathbf{B}$.

Bayes' rule helps to formulate the inference, after the acquisition of the measurement vector $\mu$ :

$$
\begin{aligned}
p(\boldsymbol{\sigma} \mid \boldsymbol{\mu})= & \frac{p(\boldsymbol{\mu} \mid \boldsymbol{\sigma}) p(\boldsymbol{\sigma})}{p(\boldsymbol{\mu})}=\frac{p_{e}(\boldsymbol{\mu}-\mathbf{H} \boldsymbol{\sigma}) p(\boldsymbol{\sigma})}{p(\boldsymbol{\mu})} \\
\propto & \exp \left\{-\frac{1}{2}(\boldsymbol{\mu}-\mathbf{H} \boldsymbol{\sigma})^{\mathrm{T}} \mathbf{R}^{-1}(\boldsymbol{\mu}-\mathbf{H} \boldsymbol{\sigma})\right. \\
& \left.-\frac{1}{2} \boldsymbol{\sigma}^{\mathrm{T}} \mathbf{B}^{-1} \boldsymbol{\sigma}\right\} \mathbb{I}_{\boldsymbol{\sigma} \geq \mathbf{0}},
\end{aligned}
$$

where $\mathbb{I}_{\boldsymbol{\sigma} \geq \mathbf{0}}$ is equal to 1 when $\sigma_{i} \geq 0$, for every $i \leq N$. Otherwise its value is 0 .

From this inference, the source term is estimated using the maximum a posteriori estimator (MAP), denoted $\sigma_{a}$ :

$$
\sigma_{a}=\underset{\sigma}{\operatorname{argmax}} p(\boldsymbol{\sigma} \mid \boldsymbol{\mu}) .
$$

Maximising $p(\boldsymbol{\sigma} \mid \boldsymbol{\mu})$ is equivalent to maximising $\ln p(\boldsymbol{\sigma} \mid \boldsymbol{\mu})$, which is equivalent to maximising the term in the exponential under the constraint of positivity, which is ultimately equivalent to minimising cost function Eq. (3) under the constraint of positivity. Since there is no analytical solution to this problem, the positivity of $\sigma$ should be enforced during the numerical minimisation which is performed with a bounded quasiNewton algorithm (Byrd et al., 1995).

In practice, because of the low number of observations, the estimated source term is very sensitive to the matrices $\mathbf{R}$ and $\mathbf{B}$, i.e. to the hyper-parameters $\left(r_{i}\right)_{1 \leq i \leq N_{d}}$ and $m$. This is the reason why we have to estimate these parameters rigorously. We propose to extend the methods developed in Winiarek et al. (2012b) to the use of several different data sets by simultaneously estimating the respective hyper-parameters.

\subsection{Estimation of hyper-parameters}

\subsubsection{Unapproximated maximum likelihood values screening}

The estimation of the prior errors' magnitude proposed in this section relies on the maximum likelihood paradigm (Dee, 1995). As the prior probabilities depend on the hyper-parameters, the likelihood of the observation set, which can be written

$$
p(\boldsymbol{\mu} \mid \boldsymbol{\theta})=\int \mathrm{d} \boldsymbol{\sigma} p(\boldsymbol{\mu} \mid \boldsymbol{\sigma} ; \boldsymbol{\theta}) p(\boldsymbol{\sigma} \mid \boldsymbol{\theta})
$$


is a function of the hyper-parameters vector $\boldsymbol{\theta}=\left(r_{1}, \ldots, r_{N_{d}}, m\right)^{\mathrm{T}}$. The pdfs $p(\boldsymbol{\mu} \mid \boldsymbol{\sigma} ; \boldsymbol{\theta})$ and $p(\boldsymbol{\sigma} \mid \boldsymbol{\theta})$ are the prior pdfs defined by Eq. (4) and Eq. (6). If it exists, the vector $\boldsymbol{\theta}$ that maximises $p(\boldsymbol{\mu} \mid \boldsymbol{\theta})$ is the most likely vector of hyper-parameters consistent with the observation set $\boldsymbol{\mu}$.

The most direct way to estimate these optimal hyperparameters is to screen the likelihood function for a range of values of $\boldsymbol{\theta}$. With our hypothesis on the errors statistics, the covariance matrices and the first guess, the likelihood can be written:

$$
\begin{aligned}
p(\boldsymbol{\mu} \mid \boldsymbol{\theta})= & \frac{e^{-\frac{1}{2} \boldsymbol{\mu}^{\mathrm{T}}\left(\mathbf{R}+\mathbf{H B H}^{\mathrm{T}}\right)^{-1} \boldsymbol{\mu}}}{\sqrt{(2 \pi)^{d}\left|\mathbf{H B H}^{\mathrm{T}}+\mathbf{R}\right|}} \\
& \times \int_{\boldsymbol{\sigma} \geq 0} \frac{e^{-\frac{1}{2}\left(\boldsymbol{\sigma}-\boldsymbol{\sigma}_{\text {BLE }}\right)^{\mathrm{T}} \mathbf{P}_{\text {BLUE }}^{-1}\left(\boldsymbol{\sigma}-\boldsymbol{\sigma}_{\text {BLLE }}\right)}}{\sqrt{(\pi / 2)^{N} \mid \mathbf{P}_{\text {вLUE }}}} \mathrm{d} \boldsymbol{\sigma},
\end{aligned}
$$

where $\sigma_{\text {вие }}$ is the BLUE estimator, which in our case reads:

$$
\sigma_{\text {вLе }}=\mathbf{B H}^{\mathrm{T}}\left(\mathbf{R}+\mathbf{H B H}^{\mathrm{T}}\right)^{-1} \boldsymbol{\mu},
$$

and $\mathbf{P}_{\text {вие }}$ is the corresponding analysis error covariance matrix:

$$
\mathbf{P}_{\text {BLUE }}=\mathbf{B}-\mathbf{B H}^{\mathrm{T}}\left(\mathbf{R}+\mathbf{H B} \mathbf{H}^{\mathrm{T}}\right)^{-1} \mathbf{H B} .
$$

The integral term in Eq. (10) has no analytical solution, but can be numerically computed using a stochastic method, such as the GHK simulator from Hajivassiliou et al. (1996). Nevertheless, if the dimension of $\boldsymbol{\theta}$ is high, the size of the space to screen can lead to a costly computation.

For details about the calculation of the likelihood expression, and in particular the general case expression, and for details about the use of the GHK simulator to estimate the integral of a truncated normal distribution, one can refer to Winiarek et al. (2012b) and Winiarek et al. (2012a).

As a statistical consistent method, we considered it as our reference method, denoted ML in this study. Faster but approximate alternatives can nonetheless be considered and tested against this statistically consistent method.

\subsubsection{Iterative scheme à-la-Desroziers}

As an alternative to the costly computation of the likelihood, the use of an iterative scheme which would quickly converge to the maximum likelihood is relevant. In Winiarek et al. (2012b) we used such an iterative algorithm for the estimation of two hyper-parameters. This iterative algorithm was shown to converge to a fixed-point that corresponds, in the context of Gaussian statistics, to the pair of hyper-parameters of maximum likelihood. This algorithm is only an approximation in the context of semi-Gaussian statistics but it yielded acceptable, through slightly different, results. Building on Desroziers and Ivanov (2001) we propose an extension of this online tuning scheme to the simultaneous estimation of several prior errors variances. The formulae read:

$$
\begin{aligned}
m^{2} & =\frac{2 J_{b}\left(\boldsymbol{\sigma}_{a}\right)}{N-\operatorname{tr}\left(\mathbf{P}_{\text {вLUE }} \mathbf{B}^{-1}\right)}, \\
r_{i}^{2} & =\frac{2 J_{\text {oi }}\left(\boldsymbol{\sigma}_{a}\right)}{d_{i}-\operatorname{tr}\left(\mathbf{H}_{i} \mathbf{P}_{\text {вLE }} \mathbf{H}_{i}^{\mathrm{T}} \mathbf{R}_{i}^{-1}\right)},
\end{aligned}
$$

where $\mathbf{H}_{i}$ and $\mathbf{R}_{i}$ are the sub-blocks of respectively the Jacobian matrix $\mathbf{H}$ and the observation error covariance matrix $\mathbf{R}$ related to data set $i$, whose observation vector is noted $\boldsymbol{\mu}_{i} . \mathbf{P}_{\text {вие }}$ is defined by Eq. (12). $J_{b}$ and $J_{o i}$ are defined by:

$$
\begin{aligned}
J_{b}(\boldsymbol{\sigma}) & =\frac{1}{2} \boldsymbol{\sigma}^{\mathrm{T}} \boldsymbol{\sigma}, \\
J_{o i}(\boldsymbol{\sigma}) & =\frac{1}{2}\left(\boldsymbol{\mu}_{i}-\mathbf{H}_{i} \boldsymbol{\sigma}\right)^{\mathrm{T}}\left(\boldsymbol{\mu}_{i}-\mathbf{H}_{i} \boldsymbol{\sigma}\right) .
\end{aligned}
$$

The source vector $\sigma_{a}$ is obtained from the minimisation of the cost function:

$$
\mathcal{L}(\sigma)=\frac{J_{b}(\sigma)}{m^{2}}+\sum_{i=1}^{N_{d}} \frac{J_{o i}(\sigma)}{r_{i}^{2}}
$$

under the constraint $\boldsymbol{\sigma} \geq \mathbf{0}$. These equations can be used in an iterative scheme which we shall call Desroziers' scheme later on. This algorithm quickly converges (3-4 iterations here) to a fixed-point giving estimated hyperparameters and the related source term.

\section{Applications to the Fukushima accident}

\subsection{Observations}

In order to illustrate the proposed methods, observations from three different data sets will be considered:

- The activity concentration in the air over Japan as described and referred to in Winiarek et al. (2012b). This data set contains 104 observations.

- Starting from 18 March 2011, daily measurements of deposited ${ }^{137} \mathrm{Cs}$ in 22 prefectures, which represents a total of 198 observations ${ }^{2}$.

\footnotetext{
${ }^{2}$ http://www .mext.go.jp/english/incident/1305529. htm
} 
- Measurements of total deposited ${ }^{137} \mathrm{Cs}$ in an area near the NPP (approximately $100 \mathrm{~km}$ around). Among the 2180 deposition measurements provided by the Ministry of Education, Culture, Sports, Science and Technology (MEXT) during May and June $2011^{3}$, 16 were filtered out because they are impacted by near-field effects that are not represented by larger scale ATMs, so that 2164 observations, that are distant enough from the NPP, were considered. As shown in Fig. 5(a), they are densely distributed in space, but on the downside there is no scale of time in these observations.

The distribution of the observation sites led to use a mesoscale domain approximately covering Japan. Because of the spatial extension and density of the sites, and because of the frequency of the observations, the ATM needs a rather limited simulation domain with high resolution in space and in time.

\subsection{Modelling the atmospheric dispersion}

\subsubsection{Meteorological fields}

ECMWF meteorological fields with a spatial resolution of $0.25^{\circ} \times 0.25^{\circ}$ and a temporal resolution of 3 hours are too coarse to be used for our need. That is the reason why we computed mesoscale meteorological fields with the Weather Research and Forecasting (WRF) numerical model (Skamarock et al., 2008). The main objective is to obtain meteorological fields with a spatial resolution of approximately $5 \mathrm{~km}$ and a temporal resolution of $1 \mathrm{~h}$. The computed meteorological fields are inputs for the atmospheric transport model. Physical parametrisations as well as the design of simulation domains are summarised in Tab. 1 and the simulation domains are displayed in Fig. 1. One of the key feature is the use of several thousands of meteorological observations to constrain the meteorological fields through nudging techniques (Stauffer and Seaman, 1994).

As shown in Fig. 2, WRF simulations show a good ability to model the occurrence of rain episodes but have the general tendency to overestimate precipitation rates (Katata et al. (2012) observed the same behaviour using MM5). This bias is a severe drawback when looking at deposition processes as this study aims to do. To avoid overestimating wet deposition, we used ground observations of rain rates in Fukushima and Ibaraki prefectures to compute a global debiasing coefficient, that we

${ }^{3}$ http://www.mext.go.jp/b_menu/shingi/chousa/ gijyutu/017/shiryo/__icsFiles/afieldfile/2011/09/ 02/1310688_1.pdf found equal to 2.4 , by which were divided all precipitation rates computed by the WRF model. We have also tested local corrections of the precipitation fields using data assimilation techniques but we did not find them to be as robust and reliable as a simpler global debiasing correction.

\subsubsection{Atmospheric transport model}

The simulations of the dispersion of radionuclides from the Fukushima Daiichi nuclear power plant have been performed with the chemistry-transport model PoLAIR3D, the Eulerian model of the PolYPHEMus platform. It has been validated for the simulation of radionuclides transport on the European Tracer Experiment, on the Algeciras incident and on the Chernobyl accident (Quélo et al., 2007).

The model integrates the concentration field $c$ of ${ }^{137} \mathrm{Cs}$, following the transport equation

$$
\frac{\partial c}{\partial t}+\operatorname{div}(\boldsymbol{u} c)=\operatorname{div}\left(\rho \mathbf{K} \nabla\left(\frac{c}{\rho}\right)\right)-\Lambda^{\mathrm{s}} c-\Lambda^{\mathrm{d}} c+\sigma
$$

where $\rho$ is the air density, $\Lambda^{\mathrm{s}}$ is the scavenging rate, $\Lambda^{\mathrm{d}}$ represents the radioactive decay and $\sigma$ is the point-wise source. $\mathbf{K}$ is the matrix of turbulent diffusion, diagonal in practice. The vertical component is given by $K_{z}$, computed with Louis parametrisation (Louis, 1979). The horizontal component $K_{H}$ is taken constant. The boundary condition on the ground is

$$
K_{z} \nabla c \cdot \mathbf{n}=-v^{\mathrm{dep}} c
$$

where $\mathbf{n}$ is the upward oriented unitary vector, and $v^{\text {dep }}$ is the dry deposition velocity of ${ }^{137} \mathrm{Cs}$.

Two domains of simulation are considered. The finest domain of simulation is a mesoscale domain covering Japan, from $131.03^{\circ} \mathrm{E}$ to $144.53^{\circ} \mathrm{E}$ and from $30.72^{\circ} \mathrm{N}$ to $43.72^{\circ} \mathrm{N}$ with a spatial resolution of $0.05^{\circ} \times 0.05^{\circ}$. The number of grid points in this domain is $270 \times 260$. Because of the small size of the domain there is a risk of re-circulation of the plume outside the domain so that the model could fail to account for radionuclides reentries. To avoid such situation and in order to compute the boundary conditions to this domain by a nesting technique, another more extended domain of simulation with a coarser resolution is used. It covers a region from $115.03^{\circ} \mathrm{E}$ to $165.03^{\circ} \mathrm{E}$ and from $25.02^{\circ} \mathrm{N}$ to $60.02^{\circ} \mathrm{N}$ with a resolution of $0.25^{\circ} \times 0.25^{\circ}$. This configuration is displayed in Fig. 1. For both domains the PolaIR3D model is configured with 15 vertical levels ranging from 0 to $8000 \mathrm{~m}$.

Caesium-137 is modelled as monodispersed passive particulate matter with a radioactive decay of 11000 
Table 1: Configuration and physical parametrisations of the WRF model. A two-way nesting technique is used between domain 1 and domain 2.

\begin{tabular}{lcc}
\hline Domain & 1 & 2 \\
\hline Spatial resolution & $18 \mathrm{~km}$ & $6 \mathrm{~km}$ \\
Number of grid points & $340 \times 250$ & $241 \times 241$ \\
Number of vertical levels & 27 & 27 \\
Numerical time-step & $60 \mathrm{~s}$ & $20 \mathrm{~s}$ \\
Output time-step & $3600 \mathrm{~s}$ & $3600 \mathrm{~s}$ \\
\hline Planetary boundary layer & Yonsei University & Yonsei University \\
Micro-physics & Kessler & WRF Single Moment 3 \\
Cumulus physics & Grell-Devenyi & Grell-Devenyi \\
Longwave radiation & RRTM & RRTM \\
Shortwave radiation & Dudhia & Dudhia \\
Surface layer & MM5 similarity & MM5 similarity \\
Land surface & Noah LSM & Noah LSM \\
Nudging & Grid nudging & Grid nudging \\
\hline
\end{tabular}

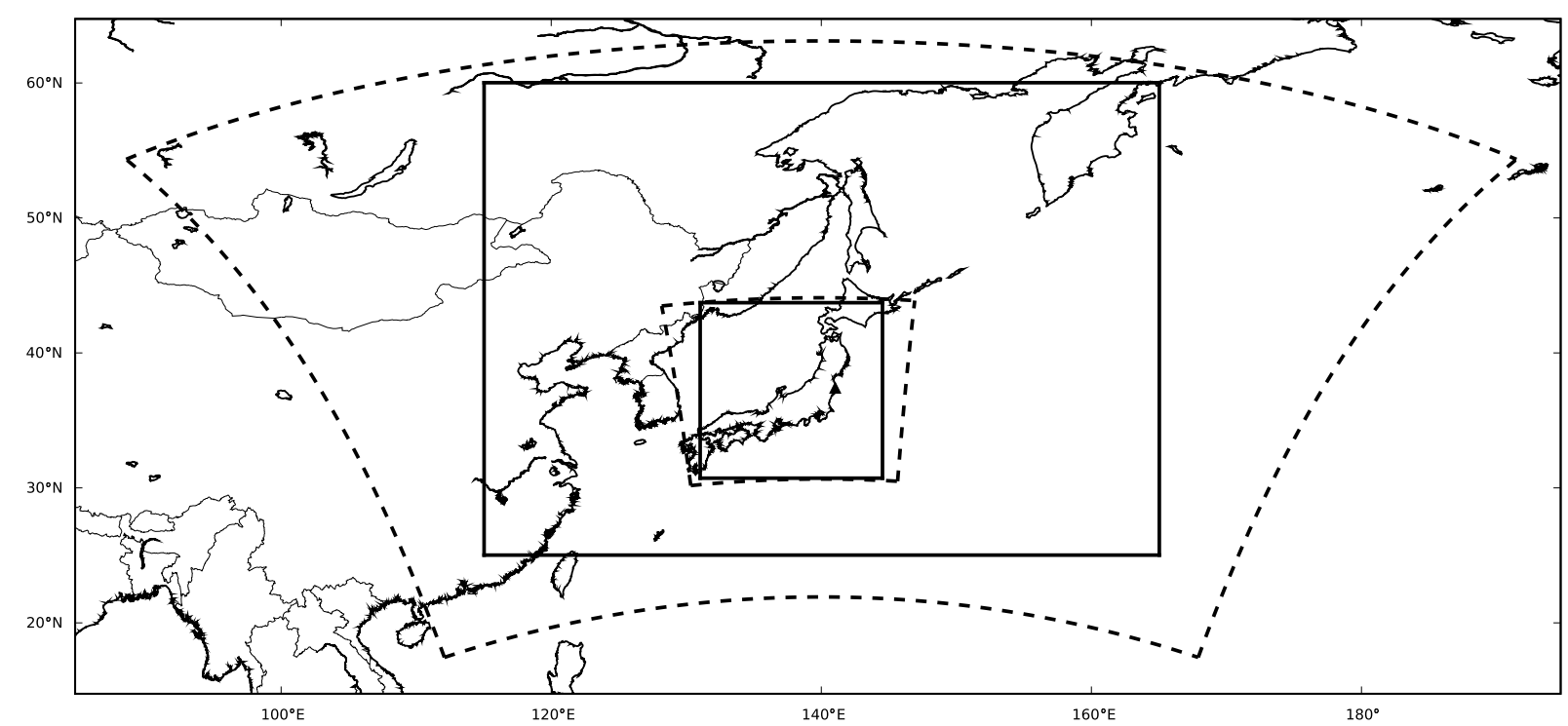

Figure 1: Map of the simulation domains used in WRF (dashed-line domains) and in PolaIRD3D (full-line domains). Two-way nesting is used in the WRF simulations and one-way nesting in the PolaIRD3D simulation. A triangle marks the location of the Fukushima Daiichi nuclear power plant. 


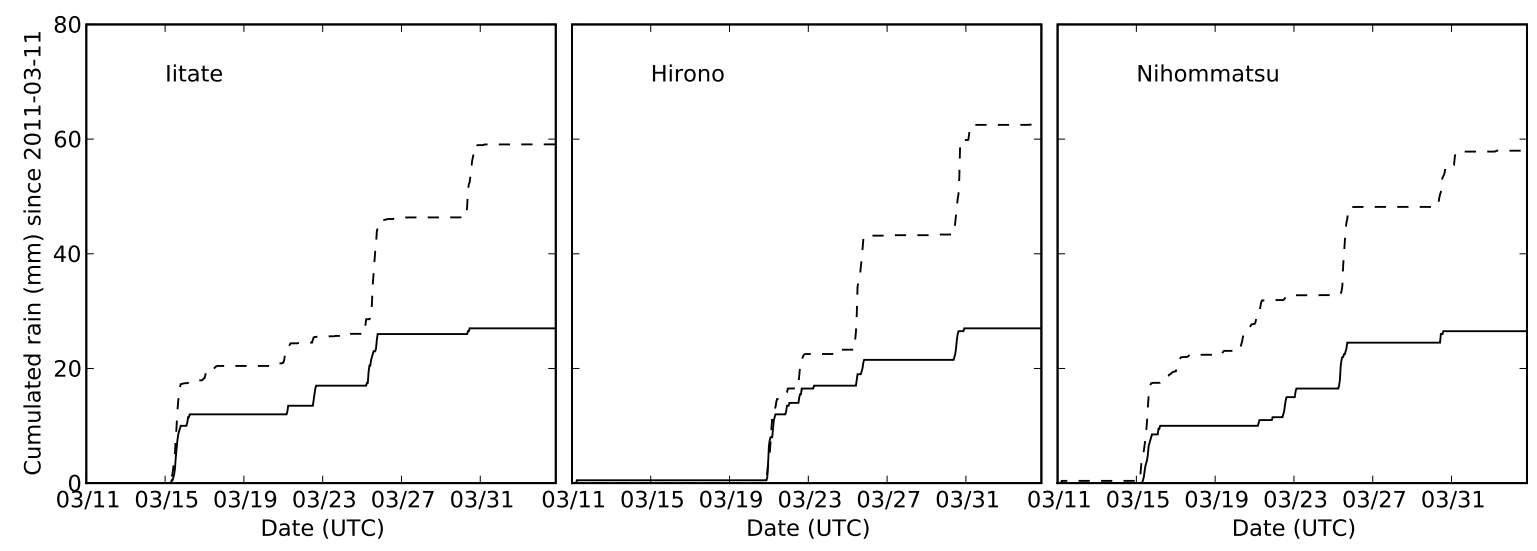

Figure 2: Comparison between WRF calculations (dashed lines) and observations (full lines) for cumulated precipitation (in mm) at some surface weather stations in Fukushima prefecture.

days. Dry deposition is modelled using a simple scheme with a constant deposition velocity: $v^{\text {dep }}=0.15 \mathrm{~cm} . \mathrm{s}^{-1}$ (Bocquet, 2012) over land, and $v^{\mathrm{dep}}=0.01 \mathrm{~cm} . \mathrm{s}^{-1}$ over the ocean (see Estournel et al. (2012) and references within). As far as wet deposition is concerned, the parametrisation used in this study is a below-cloud scavenging scheme of the form:

$$
\Lambda^{\mathrm{s}}=a\left(\frac{p}{p_{0}}\right)^{b}
$$

where $p$ stands for the precipitation rate and $p_{0}=$ $1 \mathrm{~mm} \cdot \mathrm{h}^{-1}, a$ and $b$ being two constants respectively equal to $8.410^{-5} \mathrm{~s}^{-1}$ and 0.79 following Maryon et al. (1991).

The advection is implemented thanks to a third-order direct space-time scheme, with a Koren-Sweby flux limiter function. Because of the plume sharp gradients, it is important that such a limiter is used. The diffusion scheme is integrated through an implicit second-order Rosenbrock scheme, with a three-point spatial scheme, and directional splitting.

This study attempts to reconstruct the source term from March 11 to April 1 (which represents $N=504$ one-hour time-steps). However the simulations run over a longer period (from March 11 to April 5) in order to exploit the information content of later observations. A total of 504 direct simulations are thus performed to fill the Jacobian matrix $\mathbf{H}$ column by column, and no adjoint model is needed in this process following Abida and Bocquet (2009); Winiarek et al. (2011).

\subsection{Inverse modelling results only using activity con- centrations in the air}

The proposed methods can of course be applied to inverse modelling using only one data set, such as activity concentration in the air data (the first data set described in Section 3.1 and which contains 104 observations). In this case, the formulae are rigorously equivalent to the ones presented in Winiarek et al. (2012b). It is nonetheless interesting to perform such inversion. It allows to check and confirm the consistency of the model, even though the meteorological fields and the removal processes parameterisations are different.

\subsubsection{Estimation of parameters and total released ac- tivity}

The estimated hyper-parameters and the estimated total released activities are reported in Tab. 2 together with results found in Winiarek et al. (2012b) using the same data set. The estimated hyper-parameters as well as the total released activities in the case of the ML method are close and confirm the consistency of this approach.

As discussed in Winiarek et al. (2012b) Desroziers' scheme is based on Gaussian assumptions and thus has to be considered an approximation in the case of semiGaussian assumptions for background errors statistics. In the situation where only few data are available it could yield results different from the maximum likelihood estimation. Nevertheless the gap here is smaller than it was in Winiarek et al. (2012b) with the same data. It may be caused by the use of a mesoscale model with a better resolution in space and in time which increases the general consistency in the system. The clear decrease of $r$, that represents the magnitude of the observational error, including representativeness error and 
part of model error, was to be expected and may be due to a better resolved model hence reducing the impact of model error in the inversion.

Using the maximum likelihood estimation, the total released activity of ${ }^{137} \mathrm{Cs}$ is estimated to be $1.1 \times$ $10^{16} \mathrm{~Bq}$. This is consistent with other estimations: $1.2 \times 10^{16} \mathrm{~Bq}$ for Winiarek et al. (2012b) and Chino et al. (2011), $1.3 \times 10^{16} \mathrm{~Bq}$ for Terada et al. (2012), $1.6 \times 10^{16} \mathrm{~Bq}$ for Saunier et al. (2013), $2.1 \times 10^{16} \mathrm{~Bq}$ for Mathieu et al. (2012) or $3.6 \times 10^{16} \mathrm{~Bq}$ for Stohl et al. (2012).

\subsubsection{Temporal profile and uncertainty reduction}

Due to the meteorological conditions which have often transported the radionuclides towards the Pacific Ocean, and to the low number of activity concentration data, the observability of the plume is reduced. That is why inverse modelling methods are only able to reconstruct the source term in some specific time intervals. Consequently the estimated total released activities have to be considered as lower bound estimates of the actual releases.

The reconstructed source term and its uncertainty are displayed in Fig. 3(a). The posterior uncertainty has been computed from a Monte Carlo analysis, where the observations and the background term are perturbed using their prior errors definition and the hyper-parameters estimates $\left(2 \times 10^{4}\right.$ draws and inversions are performed $)$. Then the standard deviation of the estimators ensemble is used to estimate the posterior uncertainty of the reconstructed source term. The uncertainty on the total released activity is around $65 \%$.

The time intervals of observability are clearly visible in the shape of the uncertainty. Three periods are particularly well observed: the first one lies approximately from 14 March to 15 March, the second one lies from 19 March to 22 March and the last one from 24 March to 26 March. Once again the temporal profiles of the source term and its uncertainty are very consistent with the inversion made in Winiarek et al. (2012b).

Compared to source term estimates constructed from in-situ events monitoring and core inventories (Mathieu et al., 2012), a few events are not retrieved: the first hydrogen explosions in Unit 1 on 12 March, the ventings of Unit 3 on 13 March and the events concerning Unit 2 and 3 on 16 March and 18 March. On the other hand, the multiple events of 14 March and 15 March are present in the reconstructed source term, even though in an incomplete way since the last release, probably between 7:00 UTC and 12:00 UTC on 15 March, is missing. This release is partly accounting for the north-west pattern on the deposition map, but no activity concentration observation is available to help reconstruct this event. The releases of 20 March and the ones from 21 March to 23 March are also retrieved. As far as the release around 25 March is concerned, there seems to be a slight offset of 12 hours in the reconstruction. The releases reconstructed on 19 March are not mentioned by these inventories, but seem compatible with the in-situ measurements of $\gamma$-dose rates from operator $\mathrm{TEPCO}^{4}$, for example on the northside of main office or near the west gate of the NPP. They are also retrieved by Stohl et al. (2012). They precede the attempts of emergency cooling with Tokyo Fire Department means. Finally, the releases around 30 March, not mentioned by the former inventories, are also retrieved by Terada et al. (2012).

The scatter plot of all observations is displayed in Fig. 4(a). The simulation using the source term reconstructed with activity concentration data only does not show any systematic bias when estimating the deposited activities. Again, this shows the consistency of the model and in particular of the removal processes (wet and dry deposition).

\subsection{Results of the inverse modelling using the three raw data sets}

The total deposited ${ }^{137} \mathrm{Cs}$ measurements offer no information about the time of deposition as they are measurements performed a posteriori, in May and June 2011. When only these measurements are used to attempt reconstructing the source term, the total released estimated activity seems consistent (between $1.1 \times 10^{16} \mathrm{~Bq}$ and $1.2 \times 10^{16} \mathrm{~Bq}$ ). However, the temporal profile, displayed in Fig. 3(b), is highly doubtful. Only releases on 23 and on 25 March are clearly visible. Indeed, the system has too much freedom to fit the data. The only constraint is the background term in the cost function, which in the case where the first guess is taken null (as we do) only defines a scale of amplitude. Therefore, even if these observations are abundant with a good spatial distribution, we propose in the next sections to jointly use them with other measurements with a good temporal resolution, such as activity concentrations in the air and daily measurements of deposited material. Consequently we propose to use the three data sets described in Section 3.1 in the same inversion. In this aim the prior errors have to be estimated simultaneously for the background and the three data sets $\left(N_{d}=3\right)$.

\footnotetext{
${ }^{4}$ http://www.tepco.co.jp/en/nu/monitoring/index-e. html
} 
Table 2: Estimation of parameters and corresponding reconstructed released activity for caesium-137 source reconstruction using only activity concentration observations in the air.

\begin{tabular}{llll}
\hline parameter & method & $\begin{array}{l}\text { Regional scale model } \\
\text { Winiarek et al. }(2012 \mathrm{~b})\end{array}$ & $\begin{array}{l}\text { Mesoscale model } \\
\text { This study }\end{array}$ \\
\hline \multirow{2}{*}{$r\left(\mathrm{~Bq} \mathrm{~m}^{-3}\right)$} & Desroziers' scheme & 5.4 & 2.1 \\
& Maximum likelihood & 3.3 & 1.9 \\
\hline \multirow{2}{*}{ (Bq s $\left.^{-1}\right)$} & Desroziers'scheme & $5.3 \times 10^{10}$ & $8.9 \times 10^{10}$ \\
& Maximum likelihood & $2.0 \times 10^{11}$ & $1.6 \times 10^{11}$ \\
\hline \multirow{2}{*}{ Released activity $(\mathrm{Bq})$} & Desroziers'scheme & $3.3 \times 10^{15}$ & $7.2 \times 10^{15}$ \\
& Maximum likelihood & $1.2 \times 10^{16}$ & $1.1 \times 10^{16}$ \\
\hline
\end{tabular}
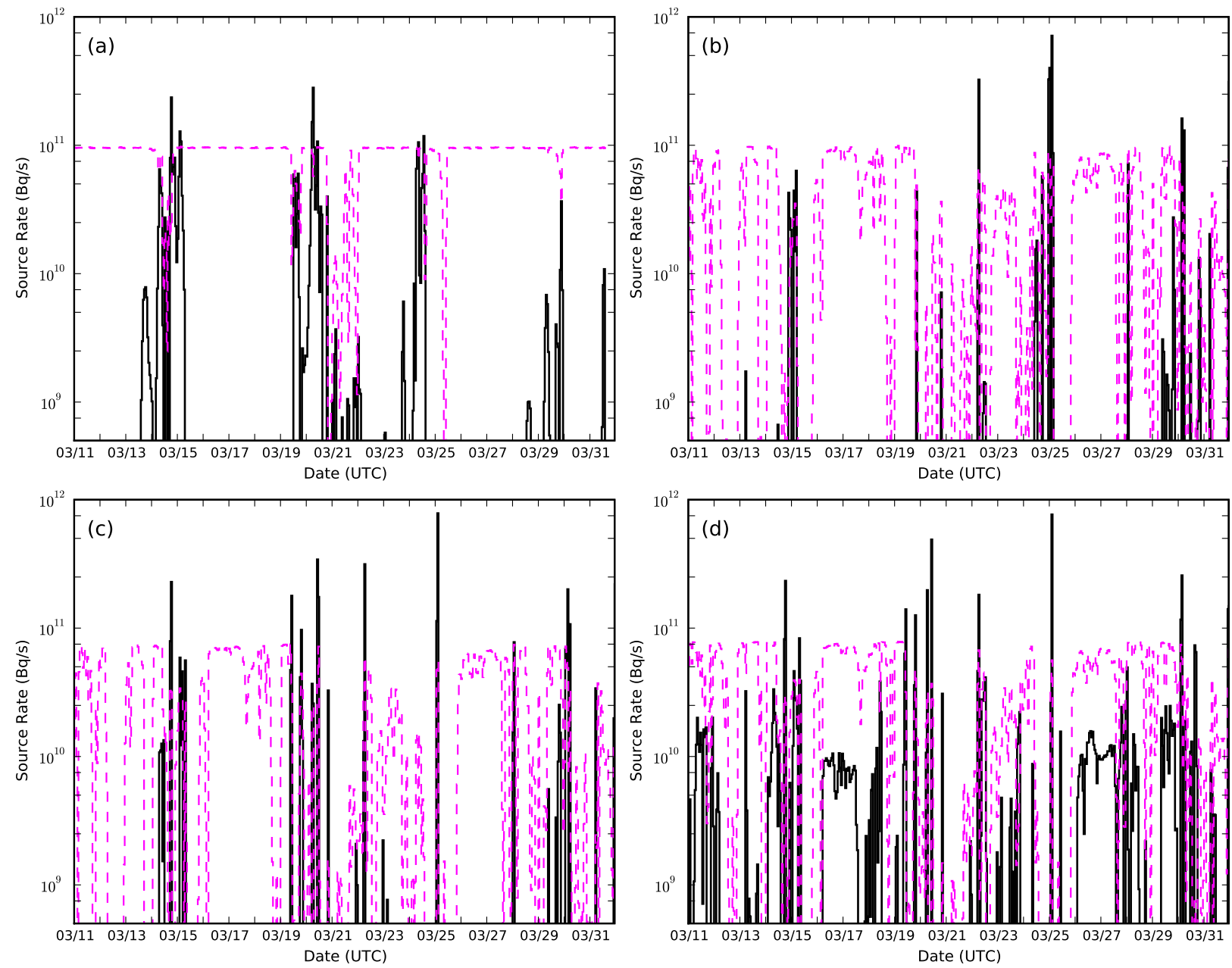

Figure 3: Full line: Temporal profile of reconstructed caesium-137 source term using (a): activity concentration of ${ }^{137} \mathrm{Cs}$ measurements in the air only, (b): total cumulated deposited ${ }^{137} \mathrm{Cs}$ measurements only, (c): the activity concentration in the air, the daily fallout measurements and the total cumulated deposition raw measurements, (d): the activity concentration in the air, the daily fallout measurements and the super-observations computed from the total cumulated deposited ${ }^{137}$ Cs. Dashed lines: posterior uncertainty of the source term computed using a Monte Carlo simulation. 

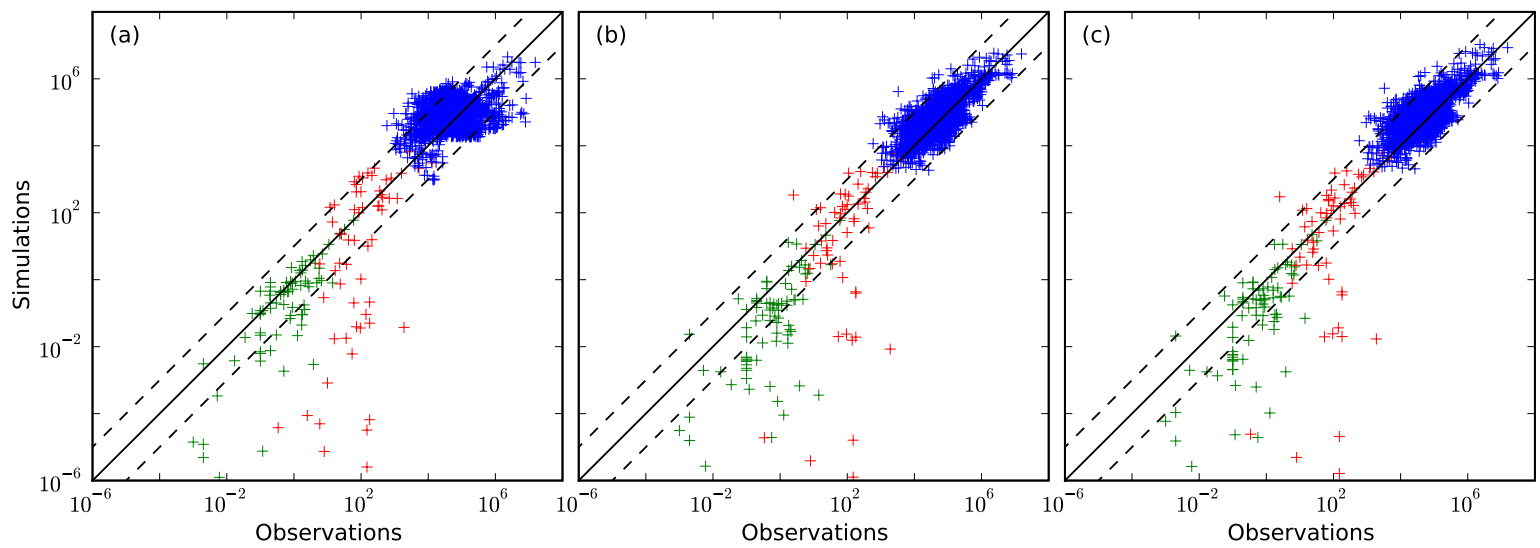

Figure 4: Scatter plots of ${ }^{137} \mathrm{Cs}$ activity concentration in the air (green crosses in $\mathrm{Bq} \mathrm{m}^{-3}$ ), daily measurements of deposited ${ }^{137} \mathrm{Cs}$ (red crosses in $\mathrm{Bq} \mathrm{m}^{-2}$ ) and total cumulated deposited ${ }^{137} \mathrm{Cs}$ (blue crosses in $\mathrm{Bq} \mathrm{m}^{-2}$ ). (a) inversion only using activity concentrations in the air. (b) inversion using the three raw data sets. (c) inversion using the three data sets including super-observations. The dashed lines represent a misfit of a factor 10 between observations and modelled values.

\subsubsection{Estimation of parameters and total released ac- tivity}

As expected, when the number of available observations increases, the results yielded by Desroziers' scheme tend to get closer to the maximum likelihood estimation.

The estimated hyper-parameters as well as the estimated total released activities are reported in Tab. 3 . Excepted for the daily measurements hyper-parameter, the values of estimated hyper-parameters do not differ much when using Desroziers' scheme or the maximum likelihood method.

Using these data sets, the total released activity of ${ }^{137} \mathrm{Cs}$ is estimated to be between $1.2 \times 10^{16} \mathrm{~Bq}$ and $1.3 \times 10^{16} \mathrm{~Bq}$.

\subsubsection{Temporal profile and uncertainty reduction}

The reconstructed source term and its uncertainty are displayed in Fig. 3(c).

The observability of the accident is improved compared to the inversion using only activity concentrations in the air. The time windows where the uncertainty is reduced are much larger, specially before 14 March and between 19 March and 26 March. The uncertainty on the total released activity has been reduced from around $65 \%$ when using only activity concentration to around $25 \%$ in this case.

The events that were retrieved in the first inversion are still reconstructed with this data set. The time offset on the release of 25 March has disappeared. The system is now able to reconstruct the late release of $15 \mathrm{March}$, from 7:00 UTC to 9:00 UTC and hence to model the north-west pattern of deposited ${ }^{137} \mathrm{Cs}$. The maps of observed and simulated total deposition are displayed in Fig. 5(a,b,c,d).

\subsection{Results of the inverse modelling using super- observations computed from the three raw data sets}

As mentioned above, measurements of the third data set are very densely distributed. This proximity certainly induces correlations in the observation errors (mainly through model errors) that are not taken into account in our system. One way to take into account these correlations would be to use a non-diagonal $\mathbf{R}$ matrix. This method would increase the computation cost as the inverse of $\mathbf{R}$ is needed for the minimisation of the cost function Eq. (3). Besides, this would introduce additional hyper-parameters in the correlation model which would have to be properly estimated as well. Such an estimation could be a difficult task in a situation with a low number of observations. Another route that can be chosen to deal with densely distributed data is the thinning of observations which consists in the optimal selection of observations. These techniques are already used in the assimilation of satellite data in the weather forecast community (Liu and Rabier, 2002). Yet another method would consist in computing super-observations by averaging all the observations contained in a model grid cell (the model resolution is $0.05^{\circ} \times 0.05^{\circ}$ ), in order to mitigate the spatial correlation in the errors. This is the method that we chose. From the 2180 measurements initially in the third data set, we computed 523 

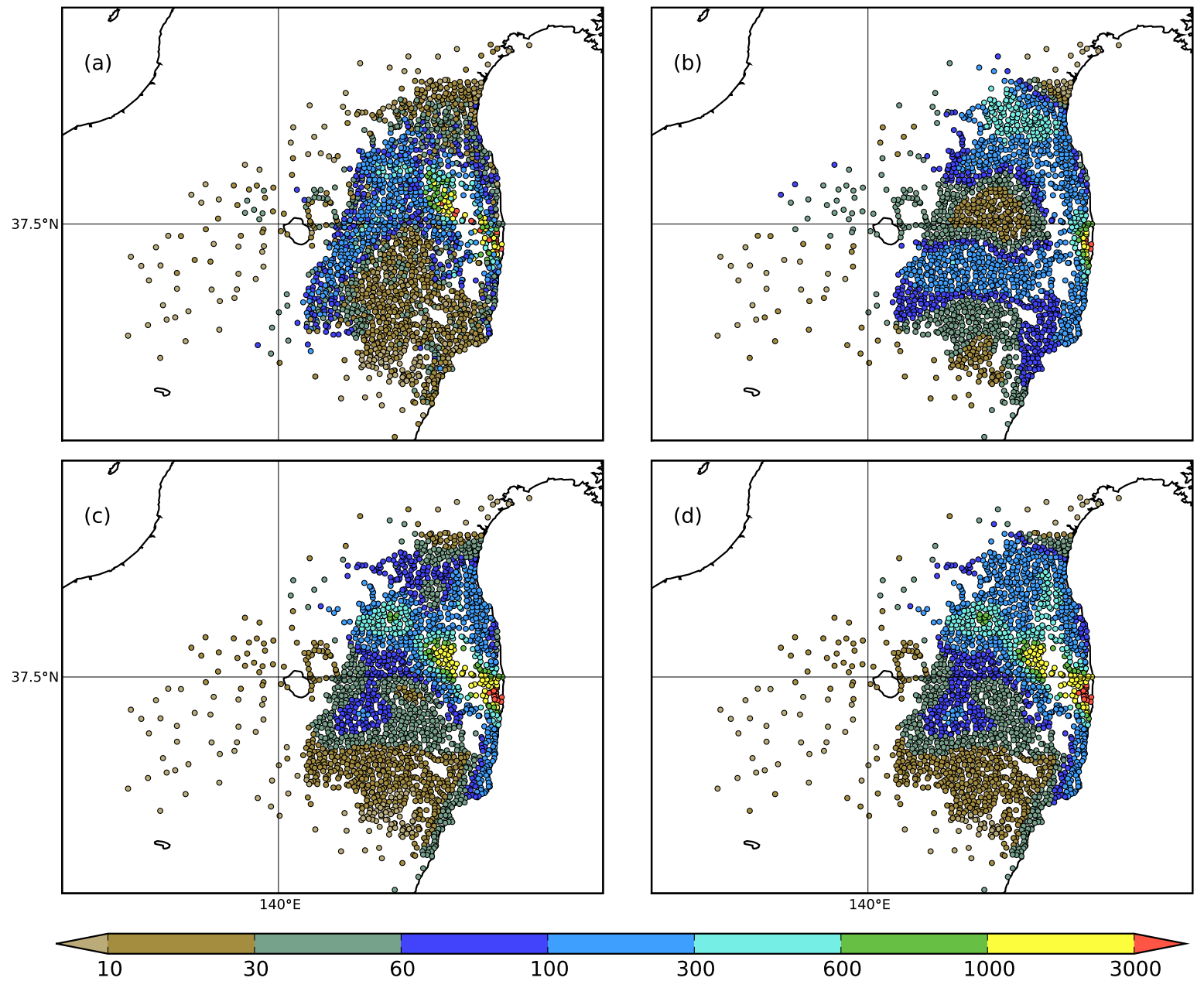

Figure 5: Map of observed and simulated deposited ${ }^{137}$ Cs. Upper left (a): measurements from MEXT. Upper right (b): simulation with source reconstructed using only ${ }^{137} \mathrm{Cs}$ activity concentrations in the air. Lower left (c): simulation with source reconstructed using ${ }^{137} \mathrm{Cs}$ activity concentrations in the air, daily measurements of deposited ${ }^{137} \mathrm{Cs}$ and measurements of total cumulated deposited ${ }^{137} \mathrm{Cs}$. Lower right (d): simulation with source reconstructed using ${ }^{137} \mathrm{Cs}$ activity concentrations in the air, daily measurements of deposited ${ }^{137} \mathrm{Cs}$ and super-observations computed from measurements of total cumulated deposited ${ }^{137} \mathrm{Cs}$. The displayed values are in $\mathrm{kBq} \mathrm{m}^{-2}$ 
Table 3: Estimation of parameters and corresponding reconstructed released activity for caesium-137 source using activity concentration, fallout daily measurements and total deposition data. $r_{1}, r_{2}$ and $r_{3}$ represents respectively the errors variances of these three data sets.

\begin{tabular}{llll}
\hline parameter & method & $\begin{array}{l}\text { with deposition } \\
\text { raw data }\end{array}$ & $\begin{array}{l}\text { with deposition } \\
\text { super-observations }\end{array}$ \\
\hline \multirow{2}{*}{$r_{1}\left(\mathrm{~Bq} \mathrm{~m}^{-3}\right)$} & Desroziers'scheme & 3.8 & 2.9 \\
& Maximum likelihood & 3.3 & 2.3 \\
\hline \multirow{2}{*}{$r_{2}\left(\mathrm{~Bq} \mathrm{~m}^{-2}\right)$} & Desroziers'scheme & 580 & 540 \\
& Maximum likelihood & 210 & 240 \\
\hline \multirow{2}{*}{$r_{3}\left(\mathrm{~Bq} \mathrm{~m}^{-2}\right)$} & Desroziers'scheme & 325000 & 240000 \\
& Maximum likelihood & 320000 & 230000 \\
\hline \multirow{2}{*}{ (Bq s$\left.^{-1}\right)$} & Desroziers'scheme & $1.2 \times 10^{11}$ & $1.3 \times 10^{11}$ \\
\hline \multirow{2}{*}{ Released activity $(\mathrm{Bq})$} & Desroziers' scheme & $1.3 \times 10^{16}$ & $1.0 \times 10^{11}$ \\
& Maximum likelihood & $1.2 \times 10^{16}$ & $1.8 \times 10^{16}$ \\
\hline
\end{tabular}

super-observations from which we eliminated 4 superobservations located too close to the NPP, hence leaving 519 super-observations to be used as the new third data set. Note that it is not necessary to estimate the reduced variance of a super-observation since this is implicitly accounted for in the related hyper-parameter estimation.

\subsubsection{Estimation of parameters and total released ac- tivity}

The estimated hyper-parameters as well as the estimated total released activities are reported in Tab. 3. As expected, the estimated standard deviation of the error in the cumulated fallout $\left(r_{3}\right)$ is significantly decreased by about $28 \%$.

Using these data sets, the total released activity of ${ }^{137} \mathrm{Cs}$ is estimated to be between $1.8 \times 10^{16} \mathrm{~Bq}$ and $1.9 \times 10^{16} \mathrm{~Bq}$.

\subsubsection{Temporal profile and uncertainty reduction}

The reconstructed source term and its uncertainty are displayed in Fig. 3(d). The uncertainty on the total released activity is estimated to be around $15 \%$ from a Monte Carlo study of $2 \times 10^{4}$ draws. However, it is the same absolute standard deviation, of about $3 \mathrm{PBq}$, as in the raw data sets inversion.

All the previously mentioned events are now retrieved with these data sets:

- Around 12 March: identified as the hydrogen explosion in Unit 1.

- On 13 March: identified as the ventings on Unit 3.

- On 14 and 15 March: multiple ventings and hydrogen explosions mainly concerning Unit 2 and
Unit 3. The late release on 15 March is now retrieved (from 7:00 to 9:00 UTC) even if its magnitude might appear weak (see section 3.6.1 for a discussion on the magnitude of the retrieved peaks).

- On 16 March: unidentified events but which correspond to pressure drops in Unit 2 and Unit 3.

- On 18 March: unidentified events probably related to Unit 3.

- On 19 March: unidentified events which correspond to an increase in several in-situ $\gamma$-dose rate measurements. The attempts of emergency cooling with the Tokyo Fire Department means began just after these events.

- On 20 March: unidentified events concerning at least Unit 2 and Unit 3.

- From 21 March to 23 March: events corresponding to smokes emitted from Unit 2 and Unit 3.

- On 25 March: unidentified event possibly concerning Unit 2. The magnitude of this peak might appear over-estimated (see section 3.6.1 for a discussion on the magnitude of the retrieved peaks).

- On 30 March: unidentified event.

From the scatter plots of Fig. 4(b,c), it is clear that the simulated cumulated deposition data are comparable when using the source term built from raw data or from super-observations. This is also visible on the deposition maps in Fig. 5(c,d). On the other hand the system's ability to simulate the two other data sets is slightly improved when using the source term retrieved from the super-observations. The assumption that the observation errors are uncorrelated led the system to give too 
much weight to the third raw data set in the inverse modelling algorithm. This over-confidence may be corrected when using super-observations so that the information content in the system is better balanced.

\subsection{Discussion about the retrieved sources}

These inversions offer an objective estimation using data assimilation techniques of what can be extracted from the data sets and the numerical model. The assimilated observations may or may not suffice to constrain the source term parameters. We showed that the cumulated deposition data alone are not sufficient to offer a satisfying chronology of the source term and that the joint assimilation of the three data sets helped to better constrain the chronology. Yet, the magnitude of the identified peaks remains questionable. The data may or may not be able to constrain them well enough, although the estimate of the total released activity seems robust.

\subsubsection{On the magnitude of the peaks}

It is generally thought that the main releases have occurred around March 15. These releases probably contributed the most to the north-west pattern of the deposition map. Our system, where the cumulated deposition data do not contain any information in time, may not be constrained enough by air concentration observations or daily measurements of fallout (specially before March 18) to precisely balance the contributions of the releases of March 14-15 (around 15\% of the total retrieved activity), March 20 (around 13\%) and March 25 (around $15 \%)$.

The peaks of March 20 and March 25 are not only reconstructed with the help of deposition measurements. They are both additionally explained by measurements of activity in the air from a particular monitoring station, located in Fukushima city, which measured an activity concentration of about $32 \mathrm{~Bq} \cdot \mathrm{m}^{-3}$ around March 20 and about $14 \mathrm{~Bq} \cdot \mathrm{m}^{-3}$ around March 25. It is possible that these measurements are caused by a re-suspension of previously deposited caesium-137. But resuspension is not implemented in our model, so that resuspension events are likely to be accounted for by fictitious releases in the source term. We tried and performed a new inversion using the same data as in Section 3.5 but without the measurements of this station. Indeed, the results from the super-observations showed an increase of the released activity on March 14 and 15, from 2.6 to 3.0 $\mathrm{PBq}$ (from $13 \%$ to $16 \%$ of an unchanged total emitted activity of $19 \mathrm{PBq}$ ), and a decrease on March 20, from 2.6 to $2.0 \mathrm{PBq}$ (from $13 \%$ to $11 \%$ of the total emitted activity). Yet, no consequences were observed on the magnitude of the peak on March 25.
We also performed inversions using a non-null first guess inferred from independent $\gamma$-dose measurements (Saunier et al., 2013), which indicates to the system that most of the releases occurred before March 18. As a result, the total estimated released activity increased by about $15 \%$, but the peak on March 25 still remained almost at the same level.

As a more drastic test and to force the system to reconstruct higher releases before March 18, we reduced the inversion window to this period. As a consequence the releases of March 15 increased (from $0.6 \mathrm{PBq}$ to $2.6 \mathrm{PBq}$ using the three raw data sets, and from $0.9 \mathrm{PBq}$ to $3.9 \mathrm{PBq}$ using the three data sets with super-observations as the third data set), but the total estimated releases decreased (respectively from $12 \mathrm{PBq}$ to $5 \mathrm{PBq}$, and from $18 \mathrm{PBq}$ to $11 \mathrm{PBq}$ ). It is consistent with the inversion using only activity concentration in the air where approximatively $6 \mathrm{PBq}$ were estimated to be released after this date. At the same time the reanalysis of deposition map has been degraded especially in the central area of the north-west pattern which is the most contaminated. This shows that: (i) considering only this shorter time window, the system can not properly reconstruct the deposition pattern shown in Fig. 5(a). (ii) Releases probably occurred after March 19 and may have contributed to the north-west pattern of the deposition map, but their magnitude is still difficult to estimate because of the lack of observations with a temporal information (such as activity concentration in the air) in this area. (iii) This may highlight the difficulty to model the removal processes and specially the wet deposition. It is also possible that the cumulated deposition map, whose measurements have been made several months after the accident, is not anymore faithful to the deposition events of the accident.

We also tested a different reconstruction resolution. Instead of reconstructing a source term with a one-hour time-step $(N=504)$, an inversion was carried out on a source term with a three-hour time-step $(N=168)$. It is generally admitted that the inversion is sensitive to the resolution of the control space (Bocquet et al., 2011) and that the system cannot generally provide reliable information at a too precise resolution (for instance the model resolution). Nevertheless, estimating the prior errors allows to compensate the impact of the control space resolution by tuning regularisation in the inversion, so that the dependence in the resolution should be mitigated. The estimated source term, using the same data sets as in Section 3.5, as well as its uncertainty are displayed in Fig. 6. The total released activity is now estimated to be $1.6 \times 10^{16} \mathrm{~Bq}$ with an uncertainty of $18 \%$. The estimated released quantities were mainly 


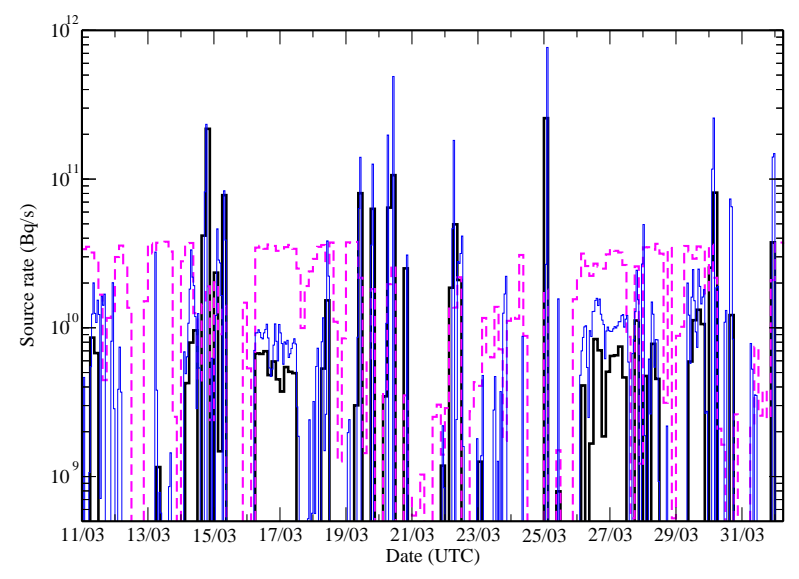

Figure 6: Full black line: Temporal profile of reconstructed caesium137 source term with a reconstruction resolution of $3 \mathrm{~h}$ using the activity concentration in the air, the daily fallout measurements and the super-observations computed from the total cumulated deposited ${ }^{137} \mathrm{Cs}$. Dashed line: posterior uncertainty of the source term computed using a Monte Carlo simulation. Thin blue line: Temporal profile of reconstructed caesium-137 source term with a reconstruction resolution of $1 \mathrm{~h}$ using the same data.

reduced on March 30 (from 2.1 PBq to $1.3 \mathrm{PBq}$ ), on March 20 (from 2.6 PBq to 2.1 PBq) and on March 25 (from $2.9 \mathrm{PBq}$ to $2.8 \mathrm{PBq}$ ), but remain at a high level. At the same time, the estimated released quantities increased on March 14-15 from 2.6 PBq to 4.1 PBq. Many of the low magnitude peaks with a high uncertainty have been reduced or have disappeared.

\subsubsection{On the difference between the sources recon- structed with raw data and super-observations}

Comparing the source profile obtained from the super-observations (Fig. 3(d)) with the source profile obtained from the inversion of the raw data sets (Fig. 3(c)), 2 PBq of caesium-137 are found to increase already existing peaks.

Moreover, when using super-observations instead of raw data, new release episodes appear in the source reconstructed term. Compared to the raw data sets inversion, $4 \mathrm{PBq}$ of caesium-137 are found in new time slots. Yet the related uncertainty is still high and even above the retrieved peaks. Nonetheless, the new releases episodes that appear in the inversion do not seem to be artefacts of the inversion algorithm. They do correspond to observed events in the NPP and to events reconstructed by other studies (Stohl et al., 2012; Saunier et al., 2013).

Judging from the $r_{i}$, the errors of the superobservations as well as their use by the model is di- agnosed as more reliable than the use of the raw data sets. By contrast, the Tikhonov regularising term is less constraining and new peaks can more easily form in the reconstructed source term.

On a physical level, the appearance of the new peaks results from the smoothing of the deposition observations that in turn leads to a smoothing in the retrieved source. This is probably why the only peak which is reduced when using super-observations is the highest one on March 25. This peak is mainly induced by the ${ }^{137} \mathrm{Cs}$ deposit observations of the north-west very thin pattern. Hence, a smoothing of this pattern can lead to a new balance of the peaks (March 15, March 20 or March 25).

\section{Conclusion}

In order to reconstruct source terms of accidental pollutant releases into the atmosphere, we have proposed an inverse modelling algorithm able to use all available data in the same inversion (concentrations in the air, measurements of fallout, integrated measurements, etc.). The algorithm relies on the relationship provided by the atmospheric transport model between the source vector and the observation set and on prior errors introduced in the system: the background errors and the observation errors. To properly balance the information content in the system a proper estimation of these prior errors is crucial.

In this aim, we proposed two methods relying on the maximum likelihood principle that we applied to the challenging reconstruction of the ${ }^{137}$ Cs source term released during the Fukushima Daiichi nuclear power plant accident in March 2011. Three data sets were used in the inversion process: activity concentrations in the air, daily measurements of fallout and total cumulated deposition data. Consequently, the prior estimation concerned 4 hyper-parameters: the variance of the background errors and the variance of each observation set errors. Averaged observations (called in the article super-observations) have also been considered for the third data set in order to reduce correlation in errors that had been neglected in the algorithm. Posterior uncertainty related to the estimated releases have also been estimated through a Monte Carlo analysis. Such an estimation is only possible with properly estimated prior errors.

With these methods and without super-observations, the total released activity is estimated to be between $1.2-1.3 \times 10^{16} \mathrm{~Bq}$ with a related uncertainty around $25 \%$. When using super-observations instead of raw fallout data, the total released activity is estimated to be between $1.8-1.9 \times 10^{16} \mathrm{~Bq}$ with a related uncertainty 
around $15 \%$. From our estimations the main ${ }^{137} \mathrm{Cs}$ contamination over Japan results from releases on 14-15 March, 19-20 March, 25 March and 30 March. Nevertheless, the uncertainty of each retrieved peak still remains high. Consequently the exact magnitude of the retrieved peaks has to be handled carefully. Some other significant releases might also have occurred when the wind was blowing directly towards the Pacific Ocean and are thus not totally reconstructed by our method using only data over Japan.

With the given data sets, the reconstruction of the source term could be improved on the condition that model error be better constrained. Two main influential sources of error were identified in the course of this study. Firstly, the reconstruction was found to be highly sensitive to the precipitation fields of the meteorological model. Even if the spatial distribution and chronology of the precipitation events were matching independent precipitation observations, we found it difficult to properly estimate the magnitude of those events. Secondly, with the given precipitation fields, the reconstruction remains sensitive to the physical process parameterisation in the ATM. One promising route to better constrain those processes is the inverse modelling of physical parameters (Bocquet, 2012).

Finally it seems promising to develop methods able to simultaneously reconstruct source terms of several radionuclides using all available data including $\gamma$-dose rates (Saunier et al., 2013). The number of observations, and in particular observations with an information of time, would then increase substantially. But the involved methods would certainly be more complex owning to correlations in observation errors and higher prior uncertainties in the data assimilation system.

\section{Acknowledgements}

This study has been supported by the IMMANENT project of Paris-Est University, and by the INSU/LEFEASSIM project ADOMOCA-2.

\section{References}

Abida, R., Bocquet, M., 2009. Targeting of observations for accidental atmospheric release monitoring. Atmos. Env. 43, 6312-6327.

Anderson, J. L., 2007. An adaptive covariance inflation error correction algorithm for ensemble filters. Tellus A 59, 210-224.

Bocquet, M., 2005. Reconstruction of an atmospheric tracer source using the principle of maximum entropy. I: Theory. Q. J. R. Meteorolog. Soc. 131, 2191-2208.

Bocquet, M., 2012. Parameter field estimation for atmospheric dispersion: Application to the Chernobyl accident using 4D-Var. Q. J. R. Meteorolog. Soc. 138, 664-681.
Bocquet, M., Pires, C. A., Wu, L., 2010. Beyond Gaussian statistical modeling in geophysical data assimilation. Mon. Wea. Rev. 138, 2997-3023.

Bocquet, M., Wu, L., Chevallier, F., 2011. Bayesian design of control space for optimal assimilation of observations. I: Consistent multiscale formalism. Q. J. R. Meteorolog. Soc. 137, 1340-1356.

Byrd, R. H., Lu, P., Nocedal, J., 1995. A limited memory algorithm for bound constrained optimization. SIAM Journal on Scientific and Statistical Computing 16, 1190-1208.

Chapnik, B., Desroziers, G., Rabier, F., Talagrand, O., 2004. Properties and first application of an error-statistics tuning method in variational assimilation. Q. J. R. Meteorolog. Soc. 130, 2253-2275.

Chapnik, B., Desroziers, G., Rabier, F., Talagrand, O., 2006. Diagnosis and tuning of observational error in a quasi-operational data assimilation setting. Q. J. R. Meteorolog. Soc. 132, 543-565.

Chino, M., Nakayama, H., Nagai, H., Terada, H., Katata, G., Yamazawa, H., 2011. Preliminary estimation of release amounts of I131 and Cs137 accidentally discharged from the Fukushima Daiichi nuclear power plant into the atmosphere. Journal of Nuclear Science and Technology 48, 1129-1134.

Davoine, X., Bocquet, M., 2007. Inverse modelling-based reconstruction of the Chernobyl source term available for long-range transport. Atmos. Chem. Phys. 7, 1549-1564.

Dee, D. P., 1995. On-line estimation of error covariance parameters for atmospheric data assimilation. Mon. Wea. Rev. 123, 11281145.

Delle Monache, L., Lundquist, J. K., Kosovic, B., Johannesson, G., Dyer, K. M., Aines, R. D., Chow, F. K., Belles, R. D., Hanley, W. G., Larsen, S. C., Loosmore, G. A., Nitao, J. J., Sugiyama, G. A., Vogt, P. J., 2008. Bayesian inference and Markov chain Monte Carlo sampling to reconstruct a contaminant source on a continental scale. Journal of Applied Meteorology and Climatology 47, 2600-2613.

Desroziers, G., Berre, L., Chapnik, B., Poli, P., 2005. Diagnosis of observation, background and analysis error statistics in observation space. Q. J. R. Meteorolog. Soc. 131, 3385-3396.

Desroziers, G., Ivanov, S., 2001. Diagnosis and adaptive tuning of observation-error parameters in a variational assimilation. Q. J. R. Meteorolog. Soc. 127, 1433-1452.

Elbern, H., Strunk, A., Schmidt, H., Talagrand, O., 2007. Emission rate and chemical state estimation by 4-dimensional variational inversion. Atmos. Chem. Phys. 7, 3749-3769.

Estournel, C., Bosc, E., Bocquet, M., Ulses, C., Marsaleix, P., Winiarek, V., Osvath, I., Nguyen, C., Duhaut, T., Lyard, F., Michaud, H., Auclair, F., 2012. Assessment of the amount of cesium-137 released to the Pacific Ocean after the Fukushima accident and analysis of its dispersion in the Japanese coastal waters. J. Geophys. Res. 117, C11014.

Hajivassiliou, V., McFadden, D., Ruud, P., 1996. Simulation of multivariate normal rectangle probabilities and their derivatives - Theoretical and computational results. Journal of Econometrics 72, 85134.

Hansen, P. C., 1992. Analysis of discrete ill-posed problems by means of the L-curve. SIAM Review 34, 561-580.

Katata, G., Terada, H., Nagai, H., Chino, M., 2012. Numerical reconstruction of high dose rate zones due to the Fukushima Dai-ichi Nuclear Power Plant accident. J. Environ. Radioactivity 111, 2-12.

Korsakissok, I., Mathieu, A., Didier, D., 2013. Atmospheric dispersion and ground deposition induced by the Fukushima Nuclear Power Plant accident: A local-scale simulation and sensitivity study. Atmos. Env. 70, 267-279.

Krysta, M., Bocquet, M., Brandt, J., 2008. Probing ETEX-II data set with inverse modelling. Atmos. Chem. Phys. 8, 3963-3971.

Li, H., Kalnay, E., Miyoshi, T., 2009. Simultaneous estimation of covariance inflation and observation errors within an ensemble 
Kalman filter. Q. J. R. Meteorolog. Soc. 135, 523-533.

Liu, Z.-Q., Rabier, F., 2002. The interaction between model resolution, observation resolution and observation density in data assimilation: A one-dimensional study. Q. J. R. Meteorolog. Soc. 128, 1367-1386.

Louis, J., 1979. A parametric model of vertical eddy fluxes in the atmosphere. Boundary-Layer Meteor. 17, 197-202.

Maryon, R. H., Smith, F. B., Conway, B. J., Goddard, D. M., 1991. The U.K. nuclear accident model. Progress in Nuclear Energy 26, 85-104.

Mathieu, A., Korsakissok, I., Quélo, D., Groëll, J., Tombette, M., Didier, D., Quentric, E., Saunier, O., Benoit, J.-P., Isnard, O., 2012. Atmospheric dispersion and deposition of radionuclides from the Fukushima Daiichi nuclear power plant accident. Elements 8, 195200.

Ménard, R., Cohn, S. E., Chang, L.-P., Lyster, P. M., 2000. Assimilation of stratospheric chemical tracer observations using a Kalman filter. Part I: Formulation. Mon. Wea. Rev. 128, 2654-2671.

Michalak, A. M., Bruhwiler, L., Tans, P. P., 2004. A geostatistical approach to surface flux estimation of atmospheric trace gases. J. Geophys. Res. 109, D14109.

Mitchell, H. L., Houtekamer, P. L., 1999. An adaptive ensemble Kalman filter. Mon. Wea. Rev. 128, 416-433.

Quélo, D., Krysta, M., Bocquet, M., Isnard, O., Minier, Y., Sportisse, B., 2007. Validation of the Polyphemus platform on the ETEX, Chernobyl and Algeciras cases. Atmos. Env. 41, 5300-5315.

Saide, P., Bocquet, M., Osses, A., Gallardo, L., 2011. Constraining surface emissions of air pollutants using inverse modeling: method intercomparison and a new two-step multiscale approach. Tellus B $63,360-370$.

Saunier, O., Mathieu, A., Didier, D., Tombette, M., Quélo, D., Winiarek, V., Bocquet, M., 2013. An inverse modeling method to assess the source term of the Fukushima nuclear power plant accident using gamma dose rate observations. Atmos. Chem. Phys. Discuss. 13, 15567-15614.

Schwinger, J., Elbern, H., 2010. Chemical state estimation for the middle atmosphere by four dimensional variational data assimilation: A posteriori validation of error statistics in observation space. J. Geophys. Res. 115, D18307.

Skamarock, W. C., Klemp, J. B., Dudhia, J., Gill, D. O., Barker, D. M., Duda, M. G., Huang, S.-Y., Wang, W., Powers, J. G., 2008. A description of the advanced research WRF version 3. Tech. rep., NCAR.

Stauffer, D. R., Seaman, N. L., 1994. On multi-scale four dimensional data assimilation. J. Appl. Meteor. 33, 416-434.

Stohl, A., Seibert, P., Wotawa, G., Arnold, D., Burkhart, J. F., Eckhardt, S., Vargas, A., Yasunari, T. J., 2012. Xenon-133 and caesium-137 releases into the atmosphere from the Fukushima Dai-ichi nuclear power plant: determination of the source term, atmospheric dispersion, and deposition. Atmos. Chem. Phys. 12, 2313-2343.

Terada, H., Katata, G., Chino, M., Nagai, H., 2012. Atmospheric discharge and dispersion of radionuclides during the Fukushima Daiichi Nuclear Power Plant accident. Part ii: verification of the source term and analysis of regional-scale atmospheric dispersion. J. Environ. Radioactivity 112, 141-154.

Winiarek, V., Bocquet, M., Saunier, O., Mathieu, A., 2012a. Correction to "Estimation of errors in the inverse modeling of accidental release of atmospheric pollutant: Application to the reconstruction of the cesium-137 and iodine-131 source terms from the Fukushima Daiichi power plant”. J. Geophys. Res. 117, D18118.

Winiarek, V., Bocquet, M., Saunier, O., Mathieu, A., 2012b. Estimation of errors in the inverse modeling of accidental release of atmospheric pollutant: Application to the reconstruction of the cesium-137 and iodine-131 source terms from the Fukushima Dai- ichi power plant. J. Geophys. Res. 117, D05122.

Winiarek, V., Vira, J., Bocquet, M., Sofiev, M., Saunier, O., 2011. Towards the operational estimation of a radiological plume using data assimilation after a radiological accidental atmospheric release. Atmos. Env. 45, 2944-2955.

Yee, E., Lien, F.-S., Keats, A., D’Amours, R., 2008. Bayesian inversion of concentration data: Source reconstruction in the adjoint representation of atmospheric diffusion. Journal of Wind Engineering and Industrial Aerodynamics 96, 1805-1816. 